\title{
Continuous Dependence on the Forchheimer Coefficient of the Forchheimer Fluid Interfacing with a Darcy Fluid
}

\author{
Jincheng Shi $(\mathbb{D})$ and Lianhong Guo \\ Huashang College Guangdong University of Finance \& Economics, Guangzhou 511300, China \\ Correspondence should be addressed to Jincheng Shi; hning0818@163.com
}

Received 15 May 2020; Accepted 23 July 2020; Published 28 August 2020

Academic Editor: Alberto Fiorenza

Copyright () 2020 Jincheng Shi and Lianhong Guo. This is an open access article distributed under the Creative Commons Attribution License, which permits unrestricted use, distribution, and reproduction in any medium, provided the original work is properly cited.

\begin{abstract}
The continuous dependence of the two fluids which interface with each other in a bounded domain is derived. It is first shown how to establish the a priori results for the solutions for the flow in a bounded domain occupied by a viscous fluid in contact with a porous solid. It is also proved that the solution depends continuously on the Forchheimer coefficient.
\end{abstract}

\section{Introduction}

In this paper, we study two fluids in one bounded domain when they interface with each other. We want to know what effect they can give to each other. Let an appropriate part of the plane $z=x_{3}=0$ denotes the boundary between a porous medium which occupies a bounded region $\Omega_{2} \subseteq \mathbb{R}^{3}$ and a nonlinear viscous fluid which occupies a bounded region $\Omega_{1} \subseteq \mathbb{R}^{3}$. The interface is denoted by $L$. The remaining parts of the boundaries of $\Omega_{1}$ and $\Omega_{2}$ are denoted, respectively, by $\Gamma_{1}$ and $\Gamma_{2}$. We also denote $\partial \Omega_{1}=\Gamma_{1} \cup L$ and $\partial \Omega_{2}=\Gamma_{2} \cup L$. We also note that $\Omega_{1}$ is above the plane $z=x_{3}=0$ and $\Omega_{2}$ is below the plane $z=x_{3}=0$.

Let $\left(u_{i}, T, p\right)$ and $\left(v_{i}, \theta, q\right)$ denote the velocity, temperature, and pressure in $\Omega_{1}$ and $\Omega_{2}$, respectively. The Forchheimer system consists of the partial differential equations (see [1])

$$
\begin{gathered}
b|\mathrm{u}| u_{i}+(1+\gamma T) u_{i}-g_{i} T+\frac{\partial p}{\partial x_{i}}=0, \quad \text { in } \Omega_{1} \times[0, \tau] \\
\frac{\partial T}{\partial t}+u_{i} \frac{\partial T}{\partial x_{i}}=\Delta T, \quad \text { in } \Omega_{1} \times[0, \tau] \\
\frac{\partial u_{i}}{\partial x_{i}}=0, \quad \text { in } \quad \Omega_{1} \times[0, \tau]
\end{gathered}
$$

where $g_{i}$ is the gravity force function. The coefficient $b$ is a positive constant which is named as the Forchheimer coefficient. The viscosity variation in (1) is accounted for by the term $1+\gamma T$, i.e., we are considering a viscosity $\mu$ like $\mu=$ $\mu_{1}(1+\gamma T), \gamma>0$.

The Darcy equations are (see Nield and Bejan [2])

$$
\begin{array}{r}
v_{i}-g_{i} \theta+\frac{\partial q}{\partial x_{i}}=0, \quad \text { in } \quad \Omega_{2} \times[0, \tau], \\
\frac{\partial \theta}{\partial t}+v_{i} \frac{\partial \theta}{\partial x_{i}}=\Delta \theta, \quad \text { in } \quad \Omega_{2} \times[0, \tau], \\
\frac{\partial v_{i}}{\partial x_{i}}=0, \quad \text { in } \quad \Omega_{2} \times[0, \tau],
\end{array}
$$

where $\Omega_{1}$ and $\Omega_{2}$ are the bounded, simply connected, and star-shaped domains and $\tau$ is a given number satisfying $0 \leq$ $\tau<\infty$. We impose the following boundary conditions:

$$
\begin{array}{cccc}
u_{i}=0 ; & T=G(x, t), & \text { on } & \Gamma_{1} \times[0, \tau], \\
v_{i} n_{i}^{(2)}=0, & \theta=\tilde{G}(x, t), & \text { on } & \Gamma_{2} \times[0, \tau],
\end{array}
$$

for prescribed functions $G(x, t)$ and $\tilde{G}(x, t)$, and $n_{i}^{(2)}$ denotes the unit outward normal of $\partial \Omega_{2}$. We also let $n_{i}^{(1)}$ be the unit 
outward normal of $\partial \Omega_{1}$. Obviously, $n_{3}^{(1)}=-n_{3}^{(2)}$ on $L$. The initial conditions are written as

$$
\begin{array}{ll}
u_{i}(x, 0)=f_{i}(x), & T(x, 0)=T_{0}(x), \quad \text { in } \Omega_{1}, \\
\theta(x, 0)=\theta_{0}(x), & \text { in } \quad \Omega_{2},
\end{array}
$$

for prescribed functions $f_{i}, T_{0}$, and $\theta_{0}$. On the interface $L$, the conditions are

$$
\begin{aligned}
u_{3} & =v_{3}, \\
T & =\theta, \\
T_{, 3} & =\theta_{, 3}, \\
q & =p .
\end{aligned}
$$

The purpose of this paper is to study the continuous dependence on the coefficients of problems (1)-(5). This type of stability is often called structural stability to distinguish it from continuous dependence on the initial data, on the boundary data, or even on the partial differential equation themselves. In continuum mechanics problems, it is necessary to be able to establish continuous dependence on the model; this is discussed in terms of differential equations by Hirsch and Smale [3]. Such stability estimates are fundamental in that one wishes to know if a small change in a coefficient in an equation or boundary data, or a small change in the equations themselves, will lead to a drastic change in the solution. When we study the continuous dependence or convergence, structural stability expresses the changes in the model itself rather than the original data. Many references to work of this nature are discussed in the monograph of Ames and Straughan [4] and the monograph of Straughan [5].

In the area of porous media, there have been many studies of continuous dependence, or structural stability, including those of Scott and Straughan [6, 7], Franchi and Straughan [8], Hoang and Ibragimov [9], Lin and Payne [10, 11], Liu $[12,13]$, Liu et al. [14-16], Scott [6], Scott and Straughan [7], Li [17], Cichon' et al. [18], Ma and Liu [19], Ciarletta et al. [20], and Payne et al. [18-21]. These results about structural stability mainly focus on one fluid in the domain. But in reality, there are usually two or more fluids in the domain. They can interface with each other. So the study of such type of problems may be interesting and meaningful. It is worth drawing attention to this type of study. Several quantitative results in physical problems were obtained in [22-25].

In this paper, we derive an a priori convergence result which compares the solution to the Forchheimer system of partial differential equations with that of the Darcy equations. The purpose of this paper is to study the continuous dependence of a solution to the Forchheimer system to a solution to Darcy equations on the Forchheimer coefficient $b$ and the effective viscosity coefficient $\gamma$. Different from $[14,26]$, there are two nonlinear terms $\gamma T u_{i}$ and $|u| u_{i}$ and there is no Laplace term in (1). So, some Sobolev inequalities do not hold for our problem. This will bring great difficulty. To get our result, we must seek a new method to overcome the difficulty. In the next section, we derive a number of a priori bounds which will be used in establishing the continuous dependence result in Section 3.

\section{A Priori Bounds}

In this section, we want to drive bounds for the various norms of $\mathrm{u}, \mathrm{v}, T$, and $\theta$.

2.1. Bounds for $\|u\|_{L_{3}\left(\Omega_{1}\right)}^{3},\|u\|_{L_{2}\left(\Omega_{1}\right)}^{2}$, and $\|v\|_{L_{2}\left(\Omega_{2}\right)}^{2}$. Multiplying $(1.1)_{1}$ with $u_{i}$ and integrating over $\Omega_{1}$, we obtain

$$
b\|u\|_{L_{3}\left(\Omega_{1}\right)}^{3}+\|\sqrt{1+\gamma T} u\|_{L_{2}\left(\Omega_{1}\right)}^{2}=\int_{\Omega_{1}} g_{i} T u_{i} d x-\int_{\Omega_{1}} \frac{\partial p}{\partial x_{i}} u_{i} d x .
$$

Integrating by parts and using Young's inequality and the arithmetic-geometric mean inequality now lead to

$$
\begin{aligned}
b\|u\|_{L_{3}\left(\Omega_{1}\right)}^{3} & +\|\sqrt{1+\gamma T} u\|_{L_{2}\left(\Omega_{1}\right)}^{2}=\int_{\Omega_{1}} g_{i} T u_{i} d x \\
& -\int_{L} p u_{3} n_{3}^{(1)} d A \leq \frac{1}{2}\|u\|_{L_{2}\left(\Omega_{1}\right)}^{2}+\frac{1}{2} g^{2}\|T\|_{L_{2}\left(\Omega_{1}\right)}^{2} \\
& +\int_{L} q v_{i} n_{i}^{(2)} d A
\end{aligned}
$$

where $g^{2}=\max g_{i} g_{i}$. By using the divergence theorem and Equation (2), we get

$$
\begin{aligned}
2 b\|u\|_{L_{3}\left(\Omega_{1}\right)}^{3} & +\|\sqrt{1+\gamma T} u\|_{L_{2}\left(\Omega_{1}\right)}^{2} \leq g^{2}\|T\|_{L_{2}\left(\Omega_{1}\right)}^{2} \\
& +2 \int_{\Omega_{2}} v_{i}\left(g_{i} \theta-v_{i}\right) d x \leq g^{2}\|T\|_{L_{2}\left(\Omega_{1}\right)}^{2} \\
& +g^{2}\|\theta\|_{L_{2}\left(\Omega_{2}\right)}^{2}-\|v\|_{L_{2}\left(\Omega_{2}\right)}^{2} .
\end{aligned}
$$

So, we have

$$
\begin{aligned}
2 b\|u\|_{L_{3}\left(\Omega_{1}\right)}^{3} & +\|\sqrt{1+\gamma T} u\|_{L_{2}\left(\Omega_{1}\right)}^{2}+\|v\|_{L_{2}\left(\Omega_{2}\right)}^{2} \leq g^{2}\|T\|_{L_{2}\left(\Omega_{1}\right)}^{2} \\
& +g^{2}\|\theta\|_{L_{2}\left(\Omega_{2}\right)}^{2} .
\end{aligned}
$$

2.2. Bounds for $\|T\|_{L_{2}\left(\Omega_{1}\right)}^{2}$ and $\|\theta\|_{L_{2}\left(\Omega_{2}\right)}^{2}$. Payne et al. [27] have obtained the following result:

$$
\sup _{[0, \tau]}\|T\|_{\infty} \leq T_{M},
$$

with $T_{M}=\max \left\{\left\|T_{0}\right\|_{\infty}, \sup _{[0, \tau]}\|G\|_{\infty}, T_{L M}\right\}$ and $T_{L M}$ is the maximum temperature on the interface $L$. Similarly, in the $\Omega_{2} \times[0, \tau]$,

$$
\sup _{[0, \tau]}\|\theta\|_{\infty} \leq \theta_{M}
$$


with $\theta_{M}=\max \left\{\left\|\theta_{0}\right\|_{\infty}, \sup _{[0, \tau}\|\tilde{G}\|_{\infty}, T_{L M}\right\}$. However, in the area $\Omega_{1} \cup \Omega_{2} \times[0, \tau]$, the maximum temperature cannot be reached on the interface $L$. Therefore, we observe that

$$
\max \left\{\sup _{[0, \tau]}\|T\|_{\infty}, \sup _{[0, \tau]}\|\theta\|_{\infty}, T_{L M}\right\} \leq N_{M},
$$

where $\quad N_{M}=\max \left\{\left\|T_{0}\right\|_{\infty}, \sup _{[0, \tau]}\|G\|_{\infty},\left\|\theta_{0}\right\|_{\infty}, \sup _{[0, \tau]}\right.$ $\left.\|\tilde{G}\|_{\infty}\right\}$. To derive the bounds for $\|T\|_{L_{2}\left(\Omega_{1}\right)}^{2}$ and $\|\theta\|_{L_{2}\left(\Omega_{2}\right)}^{2}$, we introduce another two functions $\varphi$ and $\tilde{\varphi}$ which for each $t$ satisfy

$$
\begin{gathered}
\varphi_{, \eta}-\Delta \varphi=0, \quad \text { in } \quad \Omega_{1}, \\
\tilde{\varphi}_{, \eta}-\Delta \tilde{\varphi}=0, \quad \text { in } \quad \Omega_{2}, \\
\varphi=G, \quad \text { on } \quad \Gamma_{1}, \\
\tilde{\varphi}=\tilde{G}, \quad \text { on } \quad \Gamma_{2}, \\
\varphi(x, 0)=T_{0}, \quad \text { in } \quad \Omega_{1}, \\
\tilde{\varphi}(x, 0)=\theta_{0}, \quad \text { in } \quad \Omega_{2}, \\
\varphi=\tilde{\varphi}, \quad \varphi_{, 3}=\tilde{\varphi}_{, 3}, \quad \text { on } L .
\end{gathered}
$$

Then, from the identities

$$
\begin{gathered}
\int_{0}^{t} \int_{\Omega_{1}}(T-\varphi)\left[(T-\varphi)_{, \eta}+u_{i} T_{, i}-\Delta(T-\varphi)\right] d x d \eta=0, \\
\int_{0}^{t} \int_{\Omega_{2}}(\theta-\tilde{\varphi})\left[(\theta-\tilde{\varphi})_{, \eta}+v_{i} \theta_{, i}-\Delta(\theta-\tilde{\varphi})\right] d x d \eta=0,
\end{gathered}
$$

we have

$$
\begin{aligned}
& \|T-\varphi\|_{L_{2}\left(\Omega_{1}\right)}^{2}+\|\theta-\tilde{\varphi}\|_{L_{2}\left(\Omega_{2}\right)}^{2}+2 \int_{0}^{t}\|\nabla(T-\varphi)\|_{L_{2}\left(\Omega_{1}\right)}^{2} d \eta \\
& \quad+2 \int_{0}^{t}\|\nabla(\theta-\tilde{\varphi})\|_{L_{2}\left(\Omega_{2}\right)}^{2} d \eta=2 \int_{0}^{t} \int_{\Omega_{1}}(T-\varphi)_{, i} u_{i} T d x d \eta \\
& \quad+2 \int_{0}^{t} \int_{\Omega_{2}}(\theta-\tilde{\varphi})_{, i} v_{i} \theta d x d \eta .
\end{aligned}
$$

So, (20) leads to that

$$
\begin{aligned}
& \|T-\varphi\|_{L_{2}\left(\Omega_{1}\right)}^{2}+\|\theta-\tilde{\varphi}\|_{L_{2}\left(\Omega_{2}\right)}^{2} \\
& +2 \int_{0}^{t}\|\nabla(T-\varphi)\|_{L_{2}\left(\Omega_{1}\right)}^{2} d \eta+2 \int_{0}^{t}\|\nabla(\theta-\tilde{\varphi})\|_{L_{2}\left(\Omega_{2}\right)}^{2} \\
& \cdot d \eta \leq 2 N_{M}\left(\int_{0}^{t}\|\nabla(T-\varphi)\|_{L_{2}\left(\Omega_{1}\right)}^{2} d \eta \int_{0}^{t}\|u\|_{L_{2}\left(\Omega_{1}\right)}^{2} d \eta\right)^{1 / 2} \\
& +2 N_{M}\left(\int_{0}^{t}\|\nabla(\theta-\tilde{\varphi})\|_{L_{2}\left(\Omega_{2}\right)}^{2} d \eta \int_{0}^{t}\|\mathrm{v}\|_{L_{2}\left(\Omega_{2}\right)}^{2} d \eta\right)^{1 / 2} .
\end{aligned}
$$

It follows by Lemma 1 that

$$
\|T-\varphi\|_{\Omega_{1}}^{2}+\|\theta-\tilde{\varphi}\|_{\Omega_{2}}^{2} \leq N_{M}^{2}\left(\int_{0}^{t}\|u\|_{L_{2}\left(\Omega_{1}\right)}^{2} d \eta+\int_{0}^{t}\|v\|_{L_{2}\left(\Omega_{2}\right)}^{2} d \eta\right),
$$

where

$$
\begin{gathered}
\|T-\varphi\|_{\Omega_{1}}^{2}=\|T-\varphi\|_{L_{2}\left(\Omega_{1}\right)}^{2}+\int_{0}^{t}\|\nabla(T-\varphi)\|_{L_{2}\left(\Omega_{1}\right)}^{2} d \eta, \\
\|\theta-\tilde{\varphi}\|_{\Omega_{2}}^{2}=\|\theta-\tilde{\varphi}\|_{L_{2}\left(\Omega_{2}\right)}^{2}+\int_{0}^{t}\|\nabla(\theta-\tilde{\varphi})\|_{L_{2}\left(\Omega_{2}\right)}^{2} d \eta .
\end{gathered}
$$

Since

$$
\begin{gathered}
\|T\|_{\Omega_{1}}^{2} \leq\|T-\varphi\|_{\Omega_{1}}^{2}+\|\varphi\|_{\Omega_{1}}^{2}, \\
\|\theta\|_{\Omega_{2}}^{2} \leq\|\theta-\tilde{\varphi}\|_{\Omega_{2}}^{2}+\|\tilde{\varphi}\|_{\Omega_{2}}^{2},
\end{gathered}
$$

we have

$$
\begin{aligned}
& \|T\|_{\Omega_{1}}^{2}+\|\theta\|_{\Omega_{2}}^{2} \leq N_{M}^{2}\left(\int_{0}^{t}\|u\|_{L_{2}\left(\Omega_{1}\right)}^{2} d \eta+\int_{0}^{t}\|v\|_{L_{2}\left(\Omega_{2}\right)}^{2} d \eta\right) \\
& +\|\varphi\|_{\Omega_{1}}^{2}+\|\tilde{\varphi}\|_{\Omega_{2}}^{2} .
\end{aligned}
$$

In computing the bounds for $\|\varphi\|_{\Omega_{1}}^{2}$ and $\|\tilde{\varphi}\|_{\Omega_{2}}^{2}$, we introduce another two functions $h$ and $\tilde{h}$ which for each $t$ satisfy

$$
\begin{gathered}
\Delta h=0, \quad \text { in } \quad \Omega_{1}, \\
\Delta \tilde{h}=0, \quad \text { in } \Omega_{2}, \\
h=G, \quad \text { on } \Gamma_{1}, \\
\tilde{h}=\tilde{G}, \quad \text { on } \Gamma_{2}, \\
h=\tilde{h}, \quad h_{, 3}=\tilde{h}_{, 3}, \quad \text { on } \quad L .
\end{gathered}
$$

Then, from the identities

$$
\begin{gathered}
\int_{0}^{t} \int_{\Omega_{1}}(\varphi-h)\left[(\varphi-h)_{, \eta}-\Delta(\varphi-h)+h_{, \eta}\right] d x d \eta=0, \\
\int_{0}^{t} \int_{\Omega_{2}}(\tilde{\varphi}-\tilde{h})\left[(\tilde{\varphi}-\tilde{h})_{, \eta}-\Delta(\tilde{\varphi}-\tilde{h})+\tilde{h}_{, \eta}\right] d x d \eta=0,
\end{gathered}
$$


it follows from (26) that

$$
\begin{aligned}
& \|\varphi-h\|_{L_{2}\left(\Omega_{1}\right)}^{2}+\|\tilde{\varphi}-\tilde{h}\|_{L_{2}\left(\Omega_{2}\right)}^{2}+2 \int_{0}^{t}\|\nabla(\varphi-h)\|_{L_{2}\left(\Omega_{1}\right)}^{2} d \eta \\
& \quad+2 \int_{0}^{t}\|\nabla(\tilde{\varphi}-\tilde{h})\|_{L_{2}\left(\Omega_{2}\right)}^{2} d \eta=\left\|T_{0}-h(x, 0)\right\|_{L_{2}\left(\Omega_{1}\right)}^{2} \\
& \quad+\left\|\theta_{0}-\tilde{h}(x, 0)\right\|_{L_{2}\left(\Omega_{2}\right)}^{2} \\
& \quad+2\left(\int_{0}^{t}\|\varphi-h\|_{L_{2}\left(\Omega_{1}\right)}^{2} d \eta \int_{0}^{t}\left\|h_{, \eta}\right\|_{L_{2}\left(\Omega_{1}\right)}^{2} d \eta\right)^{1 / 2} \\
& \quad+2\left(\int_{0}^{t}\|\tilde{\varphi}-\tilde{h}\|_{L_{2}\left(\Omega_{2}\right)}^{2} d \eta \int_{0}^{t}\left\|\tilde{h}_{, \eta}\right\|_{L_{2}\left(\Omega_{2}\right)}^{2} d \eta\right)^{1 / 2} .
\end{aligned}
$$

From (28), we have

$$
\begin{aligned}
\| \varphi & -h\left\|_{L_{2}\left(\Omega_{1}\right)}^{2}+\right\| \tilde{\varphi}-\tilde{h} \|_{L_{2}\left(\Omega_{2}\right)}^{2} \\
& \leq \int_{0}^{t}\left(\|\varphi-h\|_{L_{2}\left(\Omega_{1}\right)}^{2}+\|\tilde{\varphi}-\tilde{h}\|_{L_{2}\left(\Omega_{2}\right)}^{2}\right) d \eta+m_{1}(t),
\end{aligned}
$$

where

$$
\begin{aligned}
m_{1}(t)= & \left\|T_{0}-h(x, 0)\right\|_{L_{2}\left(\Omega_{1}\right)}^{2}+\left\|\theta_{0}-\tilde{h}(x, 0)\right\|_{L_{2}\left(\Omega_{2}\right)}^{2} \\
& +\int_{0}^{t}\left\|h_{, \eta}\right\|_{L_{2}\left(\Omega_{1}\right)}^{2} d \eta+\int_{0}^{t}\left\|\tilde{h}_{, \eta}\right\|_{L_{2}\left(\Omega_{2}\right)}^{2} d \eta .
\end{aligned}
$$

Upon integration of (29), we have

$\int_{0}^{t}\left(\|\varphi-h\|_{L_{2}\left(\Omega_{1}\right)}^{2}+\|\tilde{\varphi}-\tilde{h}\|_{L_{2}\left(\Omega_{2}\right)}^{2}\right) d \eta \leq \int_{0}^{t} m_{1}(\eta) e^{t-\eta} d \eta$.

Inserting (31) into (28), we get

$$
\begin{aligned}
\| \varphi & -h\left\|_{\Omega_{1}}^{2}+\right\| \tilde{\varphi}-\tilde{h}\|\leq\| T_{0}-h(x, 0) \|_{L_{2}\left(\Omega_{1}\right)}^{2} \\
& +\left\|\theta_{0}-\tilde{h}(x, 0)\right\|_{L_{2}\left(\Omega_{2}\right)}^{2}+\int_{0}^{t} m_{1}(\eta) e^{t-\eta} d \eta \\
& +\int_{0}^{t}\left\|h_{, \eta}\right\|_{L_{2}\left(\Omega_{1}\right)}^{2} d \eta+\int_{0}^{t}\left\|\tilde{h}_{, \eta}\right\|_{L_{2}\left(\Omega_{2}\right)}^{2} d \eta .
\end{aligned}
$$

So

$$
\begin{aligned}
& \|\varphi\|_{\Omega_{1}}^{2}+\|\tilde{\varphi}\|_{\Omega_{2}}^{2} \leq\|\varphi-h\|_{\Omega_{1}}^{2}+\|\tilde{\varphi}-\tilde{h}\|_{\Omega_{2}}^{2}+\|h\|_{\Omega_{1}}^{2} \\
& +\|\tilde{h}\|_{\Omega_{2}}^{2} \leq\|h\|_{\Omega_{1}}^{2}+\|\tilde{h}\|_{\Omega_{2}}^{2}+\left\|T_{0}-h(x, 0)\right\|_{L_{2}\left(\Omega_{1}\right)}^{2} \\
& +\left\|\theta_{0}-\tilde{h}(x, 0)\right\|_{L_{2}\left(\Omega_{2}\right)}^{2}+\int_{0}^{t} m_{1}(\eta) e^{t-\eta} d \eta \\
& \quad+\int_{0}^{t}\left\|h_{, \eta}\right\|_{L_{2}\left(\Omega_{1}\right)}^{2} d \eta+\int_{0}^{t}\left\|\tilde{h}_{, \eta}\right\|_{L_{2}\left(\Omega_{2}\right)}^{2} d \eta .
\end{aligned}
$$

The terms $\|h\|_{L_{2}\left(\Omega_{1}\right)}^{2}, \int_{0}^{t}\left\|h_{, \eta}\right\|_{L_{2}\left(\Omega_{1}\right)}^{2} d \eta$ as well as $\|\tilde{h}\|_{L_{2}\left(\Omega_{2}\right)}^{2}$, $\int_{0}^{t}\left\|\tilde{h}_{, \eta}\right\|_{L_{2}\left(\Omega_{2}\right)}^{2} d \eta$ may be bounded by boundary data by using a Rellich identity, cf. [6, 7]. Combining (13), (22), (24), and (33), we may have

$$
\begin{aligned}
& \|T\|_{L_{2}\left(\Omega_{1}\right)}^{2}+\|\theta\|_{L_{2}\left(\Omega_{2}\right)}^{2}+\int_{0}^{t}\|\nabla T\|_{L_{2}\left(\Omega_{1}\right)}^{2} d \eta \\
& +\int_{0}^{t}\|\nabla \theta\|_{L_{2}\left(\Omega_{2}\right)}^{2} d \eta \leq N_{M}^{2} g^{2} \int_{0}^{t}\left(\|T\|_{L_{2}\left(\Omega_{1}\right)}^{2}+\|\theta\|_{L_{2}\left(\Omega_{2}\right)}^{2}\right) \\
& \cdot d \eta+m_{2}(t),
\end{aligned}
$$

where

$$
\begin{aligned}
m_{2}(t)= & \|h\|_{\Omega_{1}}^{2}+\|\tilde{h}\|_{\Omega_{2}}^{2}+\left\|T_{0}-h(x, 0)\right\|_{L_{2}\left(\Omega_{1}\right)}^{2} \\
& +\left\|\theta_{0}-\tilde{h}(x, 0)\right\|_{L_{2}\left(\Omega_{2}\right)}^{2}+\int_{0}^{t} m_{1}(\eta) e^{t-\eta} d \eta \\
& +\int_{0}^{t}\left\|h_{, \eta}\right\|_{L_{2}\left(\Omega_{1}\right)}^{2} d \eta+\int_{0}^{t}\left\|\tilde{h}_{, \eta}\right\|_{L_{2}\left(\Omega_{2}\right)}^{2} d \eta
\end{aligned}
$$

After integrating (34), we have

$$
\int_{0}^{t}\left(\|T\|_{L_{2}\left(\Omega_{1}\right)}^{2}+\|\theta\|_{L_{2}\left(\Omega_{2}\right)}^{2}\right) d \eta \leq \int_{0}^{t} m_{2}(\eta) e^{N_{M}^{2} g^{2}(t-\eta)} d \eta .
$$

Inserting (36) back into (34), we have the following lemma.

Lemma 1. Let $\left(u_{i}, T, p\right)$ and $\left(v_{i}, \theta, q\right)$ be the solutions of (1)(5) with $T_{0}, \theta_{0}, G, \tilde{G} \in L_{\infty}$. Then,

$$
\begin{gathered}
\|T\|_{L_{2}\left(\Omega_{1}\right)}^{2}+\|\theta\|_{L_{2}\left(\Omega_{1}\right)}^{2}+\int_{0}^{t}\|\nabla T\|_{L_{2}\left(\Omega_{1}\right)}^{2} d \eta \\
+\int_{0}^{t}\|\nabla \theta\|_{L_{2}\left(\Omega_{1}\right)}^{2} d \eta \leq P_{1}(t),
\end{gathered}
$$

where

$$
P_{1}(t)=N_{M}^{2} g^{2} \int_{0}^{t} m_{2}(\eta) e^{N_{M}^{2} g^{2}(t-\eta)} d \eta+m_{2}(t)
$$
lemma.

Combining (13) and (34), we can obtain the following

Lemma 2. Let $\left(u_{i}, T, p\right)$ and $\left(v_{i}, \theta, q\right)$ be the solutions of (1)(5) with $T_{0}, \theta_{0}, G, \tilde{G} \in L_{\infty}$. Then,

$2 b\|u\|_{L_{3}\left(\Omega_{1}\right)}^{3}+\|\sqrt{1+\gamma T} u\|_{L_{2}\left(\Omega_{1}\right)}^{2}+\|v\|_{L_{2}\left(\Omega_{2}\right)}^{2} \leq g^{2} P_{1}(t) \doteq P_{2}(t)$. 


\section{Continuous Dependence on the Forchheimer Coefficient}

In this section, we want to derive an a priori estimate showing how $\left(u_{i}, T, p\right)$ and $\left(v_{i}, \theta, q\right)$ depend continuously on the Forchheimer coefficient $b$. Let $\left(u_{i}, T, p\right)$ and $\left(v_{i}, \theta, q\right)$ be solutions of (1)-(5) with $b=b_{1}$, and $\left(u_{i}^{*}, T^{*}, p^{*}\right)$ and $\left(v_{i}^{*}, \theta^{*}, q^{*}\right)$ be solutions of (1)-(5) with $b=b_{2}$, respectively. We define

$$
\begin{aligned}
& w_{i}=u_{i}-u_{i}^{*}, \\
& \Sigma=T-T^{*}, \\
& \pi=p-p^{*}, \\
& \sigma=b_{1}-b_{2}, \\
& w_{i}^{m}=v_{i}-v_{i}^{*}, \\
& \sum^{m}=\theta-\theta^{*}, \\
& \pi^{m}=q-q^{*}
\end{aligned}
$$

Then, $\left(w_{i}, \Sigma, \pi\right)$ satisfy the following equations

$$
\begin{gathered}
\sigma|u| u_{i}+b_{2}\left[u_{i}|u|-u_{i}^{*}\left|u^{*}\right|\right]+\left(1+\gamma T^{*}\right) w_{i} \\
+\gamma \Sigma u_{i}-g_{i} \Sigma+\pi_{, i}=0, \quad \text { in } \quad \Omega_{1} \times[0, \tau], \\
\frac{\partial \Sigma}{\partial t}+w_{i} T_{, i}+u_{i}^{*}(\Sigma)_{, i}=\Delta \Sigma, \quad \text { in } \Omega_{1} \times[0, \tau] \\
\frac{\partial w_{i}}{\partial x_{i}}=0, \quad \text { in } \quad \Omega_{1} \times[0, \tau]
\end{gathered}
$$

and $\left(w_{i}^{m}, \Sigma^{m}, \pi^{m}\right)$ satisfy equations

$$
\begin{aligned}
w_{i}^{m}-g_{i} \Sigma^{m}+\pi_{, i}^{m} & =0, \quad \text { in } \quad \Omega_{2} \times[0, \tau], \\
\frac{\partial \Sigma^{m}}{\partial t}+w_{i}^{m} \theta_{, i}+v_{i}^{*}\left(\Sigma^{m}\right)_{, i} & =\Delta \Sigma^{m}, \quad \text { in } \quad \Omega_{2} \times[0, \tau], \\
\frac{\partial w_{i}^{m}}{\partial x_{i}} & =0, \quad \text { in } \quad \Omega_{2} \times[0, \tau] .
\end{aligned}
$$

The boundary conditions are

$$
\begin{gathered}
w_{i}=0, \quad \sum=0, \quad \text { on } \quad \Gamma_{1} \times[0, \tau], \\
w_{i}^{m} n_{i}^{(2)} p=0, \quad \Sigma^{m}=0, \quad \text { on } \quad \Gamma_{2} \times[0, \tau] .
\end{gathered}
$$

The initial conditions can be written as

$$
\begin{aligned}
& w_{i}(x, 0)=0, \quad \Sigma(x, 0)=0, \quad \text { in } \Omega_{1}, \\
& \Sigma^{m}(x, 0)=0, \quad \text { in } \quad \Omega_{2} .
\end{aligned}
$$

The interface $L$ conditions are

$$
\begin{gathered}
w_{3}=w_{3}^{m}, \\
\Sigma=\Sigma^{m}, \\
\Sigma_{, 3}=\Sigma_{, 3}^{m}, \\
\pi^{m}=\pi .
\end{gathered}
$$

We observe for later convenience that $(4.3)_{1}$ may be rearranged as

$$
\sigma\left|u^{*}\right| u_{i}^{*}+b_{1}\left[u_{i}|u|-u_{i}^{*}\left|u^{*}\right|\right]+(1+\gamma T) w_{i}+\gamma \Sigma u_{i}^{*}-g_{i} \Sigma+\pi_{, i}=0
$$

Our main result is the following theorem.

Theorem 3. Let $\left(u_{i}, T, p\right)$ and $\left(v_{i}, \theta, q\right)$ be the the classical solutions to the initial-boundary value problem (1)-(5) corresponding to $b_{1}$, and $\left(u_{i}^{*}, T^{*}, p^{*}\right)$ and $\left(v_{i}^{*}, \theta^{*}, q^{*}\right)$ also be the the classical solutions to the initial-boundary value problem (1)-(5) but corresponding to $b_{2}$. Then, for any $t>0$, we have

$$
\left(u_{i}, T, p\right) \longrightarrow\left(u_{i}^{*}, T^{*}, p^{*}\right),\left(v_{i}, \theta, q\right) \rightarrow\left(v_{i}^{*}, \theta^{*}, q^{*}\right)
$$

as $b_{1} \longrightarrow b_{2}$. The differences of velocities satisfy

$$
\int_{0}^{t}\left(\|w\|_{L_{2}\left(\Omega_{1}\right)}^{2}+\left\|w^{m}\right\|_{L_{2}\left(\Omega_{2}\right)}^{2}\right) d \eta \leq \frac{\sigma^{2}}{b_{1} b_{2}} \int_{0}^{t} P_{2}(s) e^{N_{M}^{2} \int_{s}^{t} a_{1}(\eta) d \eta} d s .
$$

Furthermore, there is a positive function $a_{1}(t)$, given specifically in (58), such that

$$
\|\Sigma\|_{L_{2}\left(\Omega_{1}\right)}^{2}+\left\|\Sigma^{m}\right\|_{L_{2}\left(\Omega_{2}\right)}^{2} \leq \frac{\sigma^{2}}{b_{1} b_{2}} N_{M}^{2} \int_{0}^{t} P_{2}(s) e^{N_{M}^{2} \int_{s}^{t} a_{1}(\eta) d \eta} d s
$$

where $w, w^{m}, \Sigma, \Sigma^{m}$, and $\sigma$ have been defined in (40) and (41).

Proof. We begin with the identity

$$
\begin{aligned}
& \int_{\Omega_{1}}\left[\sigma|u| u_{i}+b_{2}\left[u_{i}|u|-u_{i}^{*}\left|u^{*}\right|\right]\right. \\
& \left.\quad+\left(1+\gamma T^{*}\right) w_{i}+\gamma \Sigma u_{i}-g_{i} \Sigma+\pi_{, i}\right] w_{i} d x=0 .
\end{aligned}
$$

From (51), it follows that

$$
\begin{aligned}
\frac{1}{2} b_{2} \| & \sqrt{|u|+\left|u^{*}\right|} \mathrm{w}\left\|_{L_{2}\left(\Omega_{1}\right)}^{2}+\right\| \sqrt{\left(1+\gamma T^{*}\right)} \mathrm{w} \|_{L_{2}\left(\Omega_{1}\right)}^{2} \\
= & -\int_{\Omega_{1}} \pi_{, i} w_{i} d x-\sigma \int_{\Omega_{1}}|u| u_{i} w_{i} d x-\gamma \int_{\Omega_{1}} \sum u_{i} w_{i} d x \\
& +\int_{\Omega_{1}} g_{i} w_{i} \sum d x .
\end{aligned}
$$


Using the divergence theorem, we have

$$
\begin{aligned}
\frac{1}{2} b_{2} \| & \sqrt{|u|+\left|u^{*}\right|} \mathrm{w}||_{L_{2}\left(\Omega_{1}\right)}^{2}+\|\left.\sqrt{\left(1+\gamma T^{*}\right)} \mathrm{w}\right|_{L_{2}\left(\Omega_{1}\right)} ^{2} \\
= & -\sigma \int_{\Omega_{1}}|u| u_{i} w_{i} d x-\int_{L} \pi w_{3} n_{3}^{(1)} d A-\gamma \int_{\Omega_{1}} \Sigma u_{i} w_{i} d x \\
& +\int_{\Omega_{1}} g_{i} w_{i} \Sigma d x=-\sigma \int_{\Omega_{1}}|u| u_{i} w_{i} d x+\int_{L} \pi^{m} w_{3}^{m} n_{3}^{(2)} d A \\
& -\gamma \int_{\Omega_{1}} \Sigma u_{i} w_{i} d x+\int_{\Omega_{1}} g_{i} w_{i} \Sigma d x-\sigma \int_{\Omega_{1}}|u| u_{i} w_{i} d x \\
& +\int_{\Omega_{2}}\left(-w_{i}^{m}+g_{i} \Sigma^{m}\right) w_{i}^{m} d x-\gamma \int_{\Omega_{1}} \Sigma u_{i} w_{i} d x \\
& +\int_{\Omega_{1}} g_{i} w_{i} \Sigma d x .
\end{aligned}
$$

We use the Cauchy-Schwarz inequality to have

$$
\begin{aligned}
& -\sigma \int_{\Omega_{1}}|u| u_{i} w_{i} d x \leq \sigma\|\sqrt{|u|} \mathrm{w}\|\left\|_{L_{2}\left(\Omega_{1}\right)}\right\| u \|_{L_{3}\left(\Omega_{1}\right)}^{3 / 2} \\
& \quad \leq \frac{b_{1}+b_{2}}{4}\|\sqrt{|u|} \mathrm{w}\|_{L_{2}\left(\Omega_{1}\right)}^{2}+\frac{\sigma^{2}}{2 b_{1}\left(b_{1}+b_{2}\right)} P_{2}(t) .
\end{aligned}
$$

Using Hölder's inequality and the A-G mean inequality, we have

$$
\int_{\Omega_{2}}\left(-w_{i}^{m}+g_{i} \Sigma^{m}\right) w_{i}^{m} d x \leq-\frac{1}{2}\left\|\mathrm{w}^{m}\right\|_{L_{2}\left(\Omega_{2}\right)}^{2}+\frac{1}{2} g^{2}\left\|\Sigma^{m}\right\|_{L_{2}\left(\Omega_{2}\right)}^{2} .
$$

We find that the result given in Appendix B of Liu [13] for $\|u\|_{L_{4}\left(\Omega_{1}\right)}^{2}$

$$
\|u\|_{L_{4}\left(\Omega_{1}\right)}^{2} \leq k\left[\|u\|_{L_{2}\left(\Omega_{1}\right)}^{2}+\|u\|_{L_{2}\left(\Omega_{1}\right)}^{1 / 2}\|\nabla u\|_{L_{2}\left(\Omega_{1}\right)}^{3 / 2}\right], \quad k>0
$$

or

$$
\|u\|_{L_{4}\left(\Omega_{1}\right)}^{2} \leq k\left[\left(1+\frac{\delta}{4}\right)\|u\|_{L_{2}\left(\Omega_{1}\right)}^{2}+\frac{3}{4} \delta^{-1 / 3}\|\nabla u\|_{L_{2}\left(\Omega_{1}\right)}^{2}\right], \quad k>0,
$$

for arbitrary positive constant $\delta>0$. We use this inequality for $\|\Sigma\|_{L_{4}\left(\Omega_{1}\right)}^{2}$ with $\delta=1$ and (2.30), (2.32) in (55) to get

$$
\begin{aligned}
& -\gamma \int_{\Omega_{1}} \sum u_{i} w_{i} d x \leq \gamma\|\sqrt{|u|}|w|\|_{L_{2}\left(\Omega_{1}\right)}\|\Sigma\|_{L_{4}\left(\Omega_{1}\right)}\|u\|_{L_{2}\left(\Omega_{1}\right)}^{1 / 2} \\
& \leq \frac{b_{1}+b_{2}}{4}\|\sqrt{|u|}|w|\|_{L_{2}\left(\Omega_{1}\right)}^{2}+\frac{k \gamma^{2}}{b_{1}+b_{2}} P_{2}^{1 / 2}(t)\left[\|\Sigma\|_{L_{2}\left(\Omega_{1}\right)}^{2}\right. \\
& +\|\Sigma\|_{L_{2}\left(\Omega_{1}\right)}^{1 / 2}\|\nabla \Sigma\|_{L_{2}\left(\Omega_{1}\right)}^{3 / 2} \leq \frac{b_{1}+b_{2}}{4}\|\sqrt{\mid u \|}|| w \mid\|_{L_{2}\left(\Omega_{1}\right)}^{2} \\
& \quad+\frac{3}{4} \delta_{1}\|\nabla \Sigma\|_{L_{2}\left(\Omega_{1}\right)}^{2}+\left(\frac{k \gamma^{2}}{b_{1}+b_{2}} P_{2}^{1 / 2}(t)+\frac{k^{4} P_{2}^{2}(t) \gamma^{8}}{64\left(b_{1}+b_{2}\right)^{4} \delta_{1}^{3}}\right) \\
& \quad \times\|\Sigma\|_{L_{2}\left(\Omega_{1}\right)}^{2},
\end{aligned}
$$

for arbitrary $\delta_{1}>0$. We also have

$$
\int_{\Omega_{1}} g_{i} w_{i} \Sigma d x \leq \frac{1}{2}\|w\|_{L_{2}\left(\Omega_{1}\right)}^{2}+\frac{1}{2} g^{2}\|\Sigma\|_{L_{2}\left(\Omega_{1}\right)}^{2} .
$$

Combining (53), (54), (55), (58), and (59), we obtain

$$
\begin{aligned}
& \frac{1}{2} b_{2}\left\|\sqrt{|u|+\left|u^{*}\right|} \mathrm{w}\right\|_{L_{2}\left(\Omega_{1}\right)}^{2}+\left\|\sqrt{\left(1+\gamma T^{*}\right)} \mathrm{w}\right\|_{L_{2}\left(\Omega_{1}\right)}^{2} \\
& +\frac{1}{2}\left\|w^{m}\right\|_{L_{2}\left(\Omega_{2}\right)}^{2} \leq \frac{b_{1}+b_{2}}{2}\|\sqrt{|u|} \mid \mathrm{w}\|_{L_{2}\left(\Omega_{1}\right)}^{2} \\
& +\frac{\sigma^{2}}{2 b_{1}\left(b_{1}+b_{2}\right)} P_{2}(t)+\frac{1}{2} g^{2}\left\|\Sigma^{m}\right\|_{L_{2}\left(\Omega_{2}\right)}^{2} \\
& +\left(\frac{k \gamma^{2}}{b_{1}+b_{2}} P_{2}^{1 / 2}(t)+\frac{k^{4} \gamma^{8}}{64\left(b_{1}+b_{2}\right)^{4} \delta_{1}^{3}} P_{2}^{2}(t)+\frac{1}{2} g^{2}\right) \\
& \cdot\|\Sigma\|_{L_{2}\left(\Omega_{1}\right)}^{2}+\frac{3}{4} \delta_{1}\|\nabla \Sigma\|_{L_{2}\left(\Omega_{1}\right)}^{2}+\frac{1}{2}\|\mathrm{w}\|_{L_{2}\left(\Omega_{1}\right)}^{2} .
\end{aligned}
$$
obtain

We follow a similar procedure starting from (47) to

$$
\begin{aligned}
& \frac{1}{2} b_{1}\left\|\sqrt{|u|+\left|u^{*}\right|} w\right\|_{L_{2}\left(\Omega_{1}\right)}^{2}+\|\sqrt{(1+\gamma T)} w\|_{L_{2}\left(\Omega_{1}\right)}^{2} \\
& +\frac{1}{2}\left\|w^{m}\right\|_{L_{2}\left(\Omega_{2}\right)}^{2} \leq \frac{b_{1}+b_{2}}{2}\left\|\sqrt{\left|u^{*}\right|} \mid \mathrm{w}\right\|_{L_{2}\left(\Omega_{1}\right)}^{2} \\
& +\frac{\sigma^{2}}{2 b_{2}\left(b_{1}+b_{2}\right)} P_{2}(t)+\frac{1}{2} g^{2}\left\|\Sigma^{m}\right\|_{L_{2}\left(\Omega_{2}\right)}^{2} \\
& +\left(\frac{k \gamma^{2}}{b_{1}+b_{2}} P_{2}^{1 / 2}(t)+\frac{k^{4} \gamma^{8}}{64\left(b_{1}+b_{2}\right)^{4} \delta_{1}^{3}} P_{2}^{2}(t)+\frac{1}{2} g^{2}\right) \\
& \cdot\|\Sigma\|_{L_{2}\left(\Omega_{1}\right)}^{2}+\frac{3}{4} \delta_{1}\|\nabla \Sigma\|_{L_{2}\left(\Omega_{1}\right)}^{2}+\frac{1}{2}\|\mathrm{w}\|_{L_{2}\left(\Omega_{1}\right)}^{2} .
\end{aligned}
$$

We add (60) and (61) to find

$$
\begin{aligned}
\| & \sqrt{\left(1+\gamma T+\gamma T^{*}\right)} w\left\|_{L_{2}\left(\Omega_{1}\right)}^{2}+\right\| w^{m} \|_{L_{2}\left(\Omega_{2}\right)}^{2} \\
& \leq \frac{\sigma^{2}}{2 b_{1} b_{2}} P_{2}(t)+a_{1}(t)\left(\|\Sigma\|_{L_{2}\left(\Omega_{1}\right)}^{2}+\left\|\Sigma^{m}\right\|_{L_{2}\left(\Omega_{2}\right)}^{2}\right) \\
& +\frac{3}{2} \delta_{1}\|\nabla \Sigma\|_{L_{2}\left(\Omega_{1}\right)}^{2},
\end{aligned}
$$


where

$$
a_{1}(t)=\frac{2 k \gamma^{2}}{b_{1}+b_{2}} P_{2}^{1 / 2}(t)+\frac{k^{4} \gamma^{8}}{32\left(b_{1}+b_{2}\right)^{4} \delta_{1}^{3}} P_{2}^{2}(t)+g^{2} .
$$

To bound $\|\Sigma\|_{L_{2}\left(\Omega_{1}\right)}^{2}$ and $\left\|\Sigma^{m}\right\|_{L_{2}\left(\Omega_{2}\right)}^{2}$, we multiply $(3.3)_{2}$ and $(3.4)_{2}$ by $\Sigma$ and $\Sigma^{m}$, respectively, and integrate by parts to find

$$
\begin{aligned}
& {\left[\|\Sigma\|_{L_{2}\left(\Omega_{1}\right)}^{2}+\left\|\Sigma^{m}\right\|_{L_{2}\left(\Omega_{2}\right)}^{2}\right]} \\
& =-2 \int_{0}^{t}\|\nabla \Sigma\|_{L_{2}\left(\Omega_{1}\right)}^{2} d \eta-2 \int_{0}^{t}\left\|\nabla \Sigma^{m}\right\|_{L_{2}\left(\Omega_{2}\right)}^{2} d \eta \\
& \quad+2 \int_{0}^{t} \int_{\Omega_{1}} w_{i} T \Sigma_{, i} d x d \eta+2 \int_{0}^{t} \int_{\Omega_{2}} w_{i}^{m} \theta \Sigma_{, i}^{m} d x d \eta \\
& \leq-\int_{0}^{t}\|\nabla \Sigma\|_{L_{2}\left(\Omega_{1}\right)}^{2} d \eta-\int_{0}^{t}\left\|\nabla \Sigma^{m}\right\|_{L_{2}\left(\Omega_{2}\right)}^{2} d \eta \\
& \quad+N_{M}^{2} \int_{0}^{t}\|w\|_{L_{2}\left(\Omega_{1}\right)}^{2} d \eta+N_{M}^{2} \int_{0}^{t}\left\|w^{m}\right\|_{L_{2}\left(\Omega_{2}\right)}^{2} d \eta,
\end{aligned}
$$

or

$$
\begin{aligned}
& \|\Sigma\|_{L_{2}\left(\Omega_{1}\right)}^{2}+\left\|\Sigma^{m}\right\|_{L_{2}\left(\Omega_{2}\right)}^{2}+\int_{0}^{t}\|\nabla \Sigma\|_{L_{2}\left(\Omega_{1}\right)}^{2} d \eta \\
& +\int_{0}^{t}\left\|\nabla \Sigma^{m}\right\|_{L_{2}\left(\Omega_{2}\right)}^{2} d \eta \leq N_{M}^{2} \int_{0}^{t}\|\mathrm{w}\|_{L_{2}\left(\Omega_{1}\right)}^{2} d \eta \\
& \quad+N_{M}^{2} \int_{0}^{t}\left\|w^{m}\right\|_{L_{2}\left(\Omega_{2}\right)}^{2} d \eta .
\end{aligned}
$$

Combining (62) and (4.26), we find that

$$
\begin{gathered}
\|w\|_{L_{2}\left(\Omega_{1}\right)}^{2}+\left\|w^{m}\right\|_{L_{2}\left(\Omega_{2}\right)}^{2} \leq \frac{\sigma^{2}}{2 b_{1} b_{2}} P_{2}(t)+\frac{3}{2} \delta_{1}\|\nabla \Sigma\|_{L_{2}\left(\Omega_{1}\right)}^{2} \\
+a_{1}(t) N_{M}^{2}\left(\int_{0}^{t}\|\mathrm{w}\|_{L_{2}\left(\Omega_{1}\right)}^{2} d \eta+\int_{0}^{t}\left\|\mathrm{w}^{m}\right\|_{L_{2}\left(\Omega_{2}\right)}^{2} d \eta\right)
\end{gathered}
$$

or

$$
\check{\Xi}^{\prime}(t) \leq \frac{\sigma^{2}}{2 b_{1} b_{2}} P_{2}(t)+\frac{3}{2} \delta_{1}\|\nabla \Sigma\|_{L_{2}\left(\Omega_{1}\right)}^{2}+a_{1}(t) N_{M}^{2} \sqsubseteq(t),
$$

where

$$
\sqsubseteq(t)=\int_{0}^{t}\|w\|_{L_{2}\left(\Omega_{1}\right)}^{2} d \eta+\int_{0}^{t}\left\|w^{m}\right\|_{L_{2}\left(\Omega_{2}\right)}^{2} d \eta .
$$

Thus, after integration, we may derive from (67) the estimate:

$$
\begin{aligned}
\sqsubseteq(t) & \leq \frac{\sigma^{2}}{2 b_{1} b_{2}} \int_{0}^{t} P_{2}(s) e^{N_{M}^{2} \int_{s}^{t} a_{1}(\eta) d \eta} d s \\
& +\frac{3}{2} \delta_{1} e^{N_{M}^{2} \int_{0}^{\tau} a_{1}(\eta) d \eta} \int_{0}^{t}\|\nabla \Sigma\|_{L_{2}\left(\Omega_{1}\right)}^{2} d \eta .
\end{aligned}
$$

Using (65) and (66) and choosing $\delta_{1}$ in (69), we have

$$
\begin{gathered}
\int_{0}^{t}\|\mathrm{w}\|_{L_{2}\left(\Omega_{1}\right)}^{2} d \eta+\int_{0}^{t}\left\|w^{m}\right\|_{L_{2}\left(\Omega_{2}\right)}^{2} d \eta \leq \frac{\sigma^{2}}{b_{1} b_{2}} \int_{0}^{t} P_{2}(s) e^{N_{M}^{2} \int_{s}^{t} a_{1}(\eta) d \eta} d s, \\
\|\Sigma\|_{L_{2}\left(\Omega_{1}\right)}^{2}+\left\|\Sigma^{m}\right\|_{L_{2}\left(\Omega_{2}\right)}^{2} \leq \frac{\sigma^{2}}{b_{1} b_{2}} N_{M}^{2} \int_{0}^{t} P_{2}(s) e^{N_{M}^{2} \int_{s}^{t} a_{1}(\eta) d \eta} d s .
\end{gathered}
$$

Inequalities (70) and (71) are a priori bounds demonstrating continuous dependence of the solution on the Forchheimer coefficient $b$.

\section{Data Availability}

All data generated or analyzed during this study are included within the article.

\section{Conflicts of Interest}

The authors declare that there are no conflicts of interest.

\section{Acknowledgments}

This work is supported by the Key Projects of Universities in Guangdong Province (Natural Science) (2019KZDXM042).

\section{References}

[1] L. E. Payne, J. C. Song, and B. Straughan, "Continuous dependence and convergence results for Brinkman and Forchheimer models with variable viscosity," Proceedings of the Royal Society A: Mathematical, Physical and Engineering Sciences, vol. 455, no. 1986, pp. 2173-2190, 1999.

[2] D. A. Nield and A. Bejan, Convection in Porous Media, Springer-Verlag, New York, NY, USA, 1992.

[3] M. W. Hirsch and S. Smale, Differential Equations, Dynamical Systems, and Linear Algebra, Academic Press, NewYork, 1974.

[4] K. A. Ames and B. Straughan, "Some further improperly posed problems," Mathematics in Science and Engineering, vol. 194, 1997.

[5] B. Straughan, "The Energy Method, Stability and Nonlinear Convection," Applied Mathematical Sciences, vol. 91, 2004.

[6] N. L. Scott, "Continuous dependence on boundary reaction terms in a porous medium of Darcy type," Journal of Mathematical Analysis and Applications, vol. 399, no. 2, pp. 667675, 2013.

[7] N. L. Scott and B. Straughan, "Continuous dependence on the reaction terms in porous convection with surface reactions," Quarterly of Applied Mathematics, vol. 71, no. 3, pp. 501508, 2013.

[8] F. Franchi and B. Straughan, "Continuous dependence and decay for the Forchheimer equations," Proceedings of the Royal 
Society A: Mathematical, Physical and Engineering Sciences, vol. 459, no. 2040, pp. 3195-3202, 2003.

[9] L. Hoang and A. Ibragimov, "Structural stability of generalized Forchheimer equations for compressible fluids in porous media," Nonlinearity, vol. 24, no. 1, pp. 1-41, 2011.

[10] C. Lin and L. E. Payne, "Structural stability for a Brinkman fluid," Mathematical Methods in the Applied Sciences, vol. 30, no. 5, pp. 567-578, 2007.

[11] C. Lin and L. E. Payne, "Continuous dependence on the Soret coefficient for double diffusive convection in Darcy flow," Journal of Mathematical Analysis and Applications, vol. 342, no. 1, pp. 311-325, 2008.

[12] Y. Liu, "Convergence and continuous dependence for the Brinkman-Forchheimer equations," Mathematical and Computer Modelling, vol. 49, no. 7-8, pp. 1401-1415, 2009.

[13] Y. Liu, "Continuous dependence for a thermal convection model with temperature- dependent solubility," Applied Mathematics and Computation, vol. 308, pp. 18-30, 2017.

[14] Y. Liu, S. Xiao, and Y. Lin, "Continuous dependence for the Brinkman-Forchheimer fluid interfacing with a Darcy fluid in a bounded domain," Mathematics and Computers in Simulation, vol. 150, pp. 66-82, 2018.

[15] Y. Liu, Y. Du, and C. Lin, "Convergence results for Forchheimer's equations for fluid flow in porous media," Journal of Mathematical Fluid Mechanics, vol. 12, no. 4, pp. 576-593, 2010.

[16] Y. Liu, Y. Du, and C. Lin, "Convergence and continuous dependence results for the Brinkman equations," Applied Mathematics and Computation, vol. 215, no. 12, pp. 44434455, 2010.

[17] Y. F. Li, "Convergence results on heat source for 2D viscous primitive equations of ocean dynamics," Applied Mathematics and Mechanics, vol. 41, pp. 339-352, 2020.

[18] M. Cichoń, B. Straughan, and A. Yantir, "On continuous dependence of solutions of dynamic equations," Applied Mathematics and Computation, vol. 252, pp. 473-483, 2015.

[19] H. Ma and B. Liu, "Exact controllability and continuous dependence of fractional neutral integro- differential equations with state-dependent delay," Acta Mathematica Scientia, vol. 37, no. 1, pp. 235-258, 2017.

[20] M. Ciarletta, B. Straughan, and V. Tibullo, "Structural stability for a thermal convection model with temperature-dependent solubility," Nonlinear Analysis: Real World Applications, vol. 22, pp. 34-43, 2015.

[21] L. E. Payne and B. Straughan, "Convergence and continuous dependence for the Brinkman-Forchheimer equations," Studies in Applied Mathematics, vol. 102, no. 4, pp. 419-439, 1999.

[22] Y. Qin and P. N. Kaloni, "Convective instabilities in anisotropic porous media," Studies in Applied Mathematics, vol. 91, no. 3, pp. 189-204, 1994.

[23] Y. Qin and P. N. Kaloni, "Creeping flow past a porous spherical shell," Zeitschrift für Angewandte Mathematik und Mechanik, vol. 73, no. 2, pp. 77-84, 1993.

[24] F. Franchi and B. Straughan, "Spatial decay estimates and continuous dependence on modelling for an equation from dynamo theory," Proceedings of the Royal Society A: Mathematical and Physical Sciences, vol. 445, pp. 437-451, 1994.

[25] C. Lin and L. E. Payne, "Structural stability for the Brinkman equations of flow in double diffusive convection," Journal of Mathematical Analysis and Applications, vol. 325, no. 2, pp. 1479-1490, 2007.
[26] L. E. Payne and B. Straughan, "Analysis of the boundary condition at the interface between a viscous fluid and a porous medium and related modelling questions," Journal de Mathématiques Pures et Appliquées, vol. 77, no. 4, pp. 317-354, 1998.

[27] L. E. Payne, J. F. Rodrigues, and B. Straughan, "Effect of anisotropic permeability on Darcy's law," Mathematicsl Methods in the Applied Sciences, vol. 24, no. 6, pp. 427-438, 2001. 